\title{
Structural Studies of Manganese(III) Complex with Spin-Crossover and Thermochromic Properties
}

\author{
D Villaman ${ }^{1}$, C McMonagle ${ }^{2}$, M Probert $^{2}$, O Peña ${ }^{3}$, Y Peña ${ }^{4}, M_{\text {Fuentealba }}{ }^{5}$ \\ ${ }^{1}$ Pontificia Universidad Católica de Valparaíso, ${ }^{2}$ Newcastle University, United Kingdom, ${ }^{3}$ Université \\ de Rennes-1, Rennes, France, ${ }^{4}$ Universidad Santo Tomás, Chile, ${ }^{5}$ Pontificia Universidad Católica \\ de Valparaíso, Valparaíso, Valparaíso \\ david.villaman.f@gmail.com
}

The phenomenon of spin-crossover (SCO) has been of great interest due to the bistability of the materials and the search for new technologies and applications. The transition implies a reversible change from high to low spin or vice versa[1]. This magnetic behavior can be induced by external disturbances, such as, temperature, pressure and/or light (LIESST) in $3 \mathrm{~d} 4$ to $3 \mathrm{~d} 7$ configurations. More rare and exceptional are the cases in $\mathrm{Cr}(\mathrm{II})$ and $\mathrm{Mn}(\mathrm{III})[2,3]$.

A new SCO manganese(III) complex with the formula [MnIII(5-MeO-sal-N-1-5-8-12)]Cl has been studied by X-ray diffraction, using the octahedral deviation parameters[4] $\Sigma /{ }^{\circ}\left(12\right.$ angles of $\left.90^{\circ}\right)$ and trigonal distortion $\theta /{ }^{\circ}\left(24\right.$ angles of $\left.60^{\circ}\right)$, that allow the classification of spin states. The crystallographic study was carried out at four different temperatures $(100 \mathrm{~K}, 290 \mathrm{~K}, 350 \mathrm{~K}$ and $400 \mathrm{~K}$ ) and the unit cell was analyzed at twelve different temperatures in a range of $90 \mathrm{~K}$ to $400 \mathrm{~K}$, finding large changes in the coordination sphere. At $400 \mathrm{~K}$ octahedral deviations parameters were obtained according to high spin (HS) with $59.2(4)^{\circ}$ and $149.5(7)^{\circ}$, and at $100 \mathrm{~K}$ the values are typical of low spin (LS) with $34.0(4)^{\circ}$ and $79.8(6)^{\circ}$, for $\Sigma /{ }^{\circ}$ and $\theta /{ }^{\circ}$, respectively. The magnetic susceptibility confirms a gradual transition with a $\mathrm{T} 1 / 2$ of $281 \mathrm{~K}$, according to the large changes of the unit cell between $250 \mathrm{~K}$ and $310 \mathrm{~K}$ (figure 1). The definitive evidence of the electronic transition corresponds to the thermochromism in solid state. On cooling to below $250 \mathrm{~K}$, the color begins to visually change and by $90 \mathrm{~K}$ the single crystal has turned red (figure 2) with all the electronic population in LS state (3T1g ground state). Finally, a comparison with the MnIII analogous complexes was made using the octahedral distortion parameters obtaining a trend and dispersion diagram of the reported values.

References

[1] P. Gütlich, A.B. Gaspar, Y. Garcia, Beilstein J. Org. Chem. 9 (2013) 342-391.

[2] S. Wang, W.-T. Xu, W.-R. He, S. Takaishi, Y.-H. Li, M. Yamashita, W. Huang, Dalt. Trans. 45 (2016) 5676-5688.

[3] S. Wang, Y.J. Li, F.F. Ju, W.T. Xu, K. Kagesawa, Y.H. Li, M. Yamashita, W. Huang, Dalt. Trans. 46 (2017) 1106311077.

[4] R. Pritchard, S.A. Barrett, C.A. Kilner, M.A. Halcrow, T, J. Chem. Soc. Dalt. Trans. (2008) 3159-3168.

Keywords: Spin-Crossover; Thermochromism.

Acknowledgements to the Fondecyt Project No. 1130640, Fondequip EQM120095 (Single-CrystalXRD). David Villaman thanks Conicyt Scholarship No 21151093, PUCV Internship Scholarship and Newcastle University for the support.

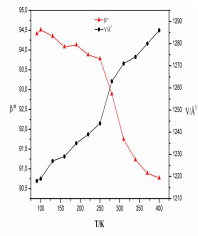

Figure 1: . $\beta$ angle (red triangles) and volume (black dots)

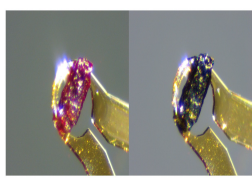

Figure 2: Thermochromism at $100 \mathrm{~K}$ and variation by temperature

Acta Cryst. (2020). A76, a192 